\title{
TU/e Em,HOEN

\section{A three-dimensional transient mixed hybrid finite element model for superabsorbent polymers with strain-dependent permeability}

\section{Citation for published version (APA):}

Yu, C., Malakpoor, K., \& Huyghe, J. M. (2018). A three-dimensional transient mixed hybrid finite element model for superabsorbent polymers with strain-dependent permeability. Soft Matter, 14(19), 3834-3848.

https://doi.org/10.1039/c7sm01587a

DOI:

10.1039/c7sm01587a

Document status and date:

Published: 16/05/2018

\section{Document Version:}

Accepted manuscript including changes made at the peer-review stage

\section{Please check the document version of this publication:}

- A submitted manuscript is the version of the article upon submission and before peer-review. There can be important differences between the submitted version and the official published version of record. People interested in the research are advised to contact the author for the final version of the publication, or visit the $\mathrm{DOI}$ to the publisher's website.

- The final author version and the galley proof are versions of the publication after peer review.

- The final published version features the final layout of the paper including the volume, issue and page numbers.

Link to publication

\footnotetext{
General rights

- You may freely distribute the URL identifying the publication in the public portal. follow below link for the End User Agreement:

www.tue.nl/taverne

\section{Take down policy}

If you believe that this document breaches copyright please contact us at:

openaccess@tue.nl

providing details and we will investigate your claim.
}

Copyright and moral rights for the publications made accessible in the public portal are retained by the authors and/or other copyright owners and it is a condition of accessing publications that users recognise and abide by the legal requirements associated with these rights.

- Users may download and print one copy of any publication from the public portal for the purpose of private study or research.

- You may not further distribute the material or use it for any profit-making activity or commercial gain

If the publication is distributed under the terms of Article $25 \mathrm{fa}$ of the Dutch Copyright Act, indicated by the "Taverne" license above, please 


\section{Journal Name}

\section{ARTICLE TYPE}

Cite this: DOI: $10.1039 /$ xxxxxxxxxx

Received Date

Accepted Date

DOI: $10.1039 / x x x x x x x x x x$

www.rsc.org/journalname

\section{A three-dimensional transient mixed hybrid finite ele- ment model for superabsorbent polymers with strain- dependent permeability ${ }^{\dagger}$}

\author{
Cong Yu, ${ }^{* a}$ Kamyar Malakpoor, ${ }^{a}$ and Jacques M.Huyghe ${ }^{a, b}$
}

\section{Introduction}

Hydrogels are hydrophilic polymers that absorb water (and therefore swell) without dissolving itself into it. During swelling, the polymer chains are extended; however, the existence of crosslinkers prevents the infinite extension of the polymer chains. As a result, hydrogels behave more like solid than fluid. The amount of water that is taken by hydrogels, based on different compositions and designs, is under the influence of external stimuli, such as temperature, $\mathrm{pH}$ and ionic strength in the aqueous solvent in contact with the polymer ${ }^{1}$.

Over the decades, the applications of hydrogels have been shown to be extensive in diverse fields ${ }^{2,3}$. In the field of biomedicine, hydrogels are widely used in drug delivery system and as scaffolds in tissue engineering ${ }^{4}$. Due to its responsive nature, hydrogels lie in the heart of the design of 'smart' biodevices and biosensors ${ }^{5}$. Their applications in agriculture have been studied as a suitable solution for holding moisture in the soil. A specific type of hydrogel, superabsorbent polymer, is the main working substance in the hygienic products (napkins and diapers).

\footnotetext{
a Mechanical engineering, Eindhoven University of Technology, Eindhoven, the Netherlands. Tel: +31 402472816; E-mail: c.yu@tue.nl

${ }^{b}$ Bernal Institute, University of Limerick, Limerick, Ireland.

$\dagger$ Electronic Supplementary Information (ESI) available: Movies of various swelling situations are included. See DOI: 10.1039/b000000x/
}

Superabsorbent polymers exhibit exceptional capability to absorb and retain a large amount of water or aqueous solutions. The water content of such a hydrogel can be up to $99 \%$ of its weight. Nowadays, taken the best performance cost ratio into consideration, industrially relevant superabsorbent polymers are mostly made from partially neutralized $\left(\mathrm{H}^{+}\right.$in the acrylic acid monomer replaced by $\mathrm{Na}^{+}$), lightly cross-linked polyacrylic acid ${ }^{6}$. They are namely sodium polyacrylate hydrogel.

Over the years, many swelling models are developed by different research groups. In general, there are two main approaches to model a swelling porous medium. One approach is based on poroelasticity founded by Biot ${ }^{7}$. Recent years, several groups are dedicated to formulating theories that apply to gels coupling mass transport and large deformation. Hong et al. ${ }^{8}$, Chester et al. ${ }^{9,10}$ and Duda et al. ${ }^{11}$ are just some examples of the vast literature. They all followed the non-equilibrium thermodynamics approach based on the Flory theory ${ }^{12,13}$ where a multiplicative kinetic relation and an addition of independent parts of Helmholtz free energy were established. The large deformation (non-linear) theory is shown to be consistent with linear poroelasticity theory in the small deformation regime ${ }^{14}$. The other approach is based on the mixture theory. This approach is usually popular among biomechanics studies due to the complexity in the composition of the biological tissues (usually multiple components coexist). This approach is first founded by Truesdell ${ }^{15}$, later further developed by Bowen ${ }^{16}$ with the application in a porous medium (multiple 
fluid phases and one solid phase). A biphasic swelling model for biological tissues is developed by Lanir ${ }^{17}$, in which the instant equilibrium of the ion phase is assumed. Lai et al. ${ }^{18}$ introduced a triphasic theory (fluid, solid and ion phase) for the swelling articular cartilage. Further, a separate consideration of cation and anions leads to a quadriphasic model ${ }^{19} \cdot \mathrm{Li}^{20}$ proposed a multieffect-coupling-ionic-strength-stimulus model in which the influence of the initial fixed charge density on the gel responses was investigated.

A numerical method that takes the underlying physical motivations into account yields more accurate quantitative results. It has been observed that during transient swelling, the volumetric expansion of the gel is strongly inhomogeneous ${ }^{21}$. This is explained by the increasing porosity (as a result of large strain) near the outer layer as the outer layer swells earlier than the core. Assuming water transporting inside the gel according to Darcy's law, the non-constant permeability tensor across the domain demands careful numerical treatment. One of the most distinctive properties possessed by MHFEM is local mass conservation. Mosé et al. ${ }^{22}$ and Kaasschieter ${ }^{23}$ reported that such a property gave a more accurate calculation of flux in the potential problems for a domain with heterogeneous permeability. Since the amount of net flux across one element has a direct influence on the volumetric change of the element in hydrogel swelling simulation, an accurate calculation of flux is also of great importance. Malakpoor et al. ${ }^{24}$ applied MHFEM in the simulation of the swelling of cartilaginous tissues and achieved good agreement with the analytical solutions in small deformation regime. To our knowledge, there is no work available yet that enables a full $3 \mathrm{D}$ simulation of a swelling hydrogel using MHFEM.

Instabilities are commonplace in our daily life, for example, wavy patterns of the leaves ${ }^{25}$ and wrinkling of the human skin. People used to avoid instabilities and study them as failure mechanics. However, thanks to the recent advances in the material science and manufacturing technologies, the buckling instabilities of soft materials (such as elastomers and hydrogels) are exploited, leading to many interesting applications 26,27 .

Many studies have been dedicated to understanding the onset of the instabilities (typically wrinkling and creasing) and pattern formation of hydrogels induced by swelling. A pioneer work is done by Tanaka et al. ${ }^{28}$, who reported transient surface instability of a spherical ionized acrylamide gel during the free swelling experiment. According to Tanaka et al. ${ }^{28}$, the cause for the surface instability is anisotropic osmotic pressure along the gel radius. As the outer layer swells first, the inner core is still relatively stiff and therefore a compressive force arises leading to the buckling of the outer surface. Later, Bertrand et al. ${ }^{21}$ also reported the transient appearance of lobes during free swelling of a spherical poly-acrylamide hydrogel as the consequence of initial smallscale roughness at the surface and the existence of compressive (azimuthal) stress.

Besides spherical gels, the buckling behavior of thin gel films fixed on a substrate has also been the center of the study from both theoretical and experimental point of view. As a matter of fact, the wrinkling or creasing of the film is caused by the same mechanism: excessive compressive stress due to geometric con- straints. Several studies applied linear perturbation analysis to study the onset of the surface instability in the quasi-static state under various situations ${ }^{29-31}$. A point perturbation method ${ }^{26}$ was introduced to analyze crease instability which is beyond linear stability analysis. Experimental work of Trujillo et al. ${ }^{32}$ suggests a compressive strain of 0.33 irrespective of the gel thickness for surface-attached polyacrylamide polymer hydrogels with a wide range of shear moduli (600 Pa-24 kPa). Guvendiren et al. ${ }^{33}$ reported that a critical expansion ratio of 1.12 for pattern formation of PHEMA hydrogel films with a depth-wise crosslinking gradient. By means of simulation, Toh et al. ${ }^{34}$ studied the evolution of wrinkles. Kang et al. ${ }^{35}$ reported the appearance of wrinkles without external perturbation in a finite element swelling under geometric constraint simulation.

In the current study, first different aspects of the swelling simulations of the superabsorbent polymer are presented. Next, a surface instability study is presented. The remainder of this paper is organized as follows. In section 2 , we introduce our physicallymotivated poromechanical swelling model. Numerical aspects of the MHFEM simulations and several numerical examples are discussed in section 3. Finally, swelling-induced surface instability is simulated. Simulation observations together with linear perturbation analysis are presented in section 4. At last, conclusions are drawn in section 5 .

\section{Poromechanical swelling model}

Although gels are not a porous media in a traditional sense, we take a poromechanical approach to model the swelling of a hydrogel. In poromechanics, the porous material mostly consists of two phases: solid skeleton and pore fluid. The gel as a whole is treated as the solid skeleton and the pore fluid refers to the water that flows into/through the gel. Our model follows the two assumptions made for the ideal elastomeric gels ${ }^{36}$. Firstly, the total volume of the gel is the sum of the dry polymer and the fluid absorbed. The condition is also known as molecular incompressibility. It basically expresses that the polymer chain and the permeating fluid molecules are incompressible. Secondly, the free energy of the gel is the sum of three parts: stretching of the network, mixing between solvent and polymer and the energy related to ionic osmosis.

\subsection{Lagrangian formulation}

There are two reference frames in continuum mechanics available: Lagrangian and Eulerian frames. In the Lagrangian frame, the observer is attached to a material particle; while in the Eulerian one, the observer is fixed in the geometric space. As a result, in solid mechanics problems, Lagrangian frame is a natural choice and in fluid mechanics, Eulerian frame is the traditional choice $^{37}$. In this study, the displacement of the solid skeleton is of primary interest; therefore, the Lagrangian frame is chosen. In other words, the observer is fixed to the solid phase. Consequently, nominal variables (variables that are defined in reference to the initial configuration) are adopted to describe the system.

One of the important concepts in poroelasticity is porosity. It refers to the local fluid volume fraction $\phi_{f}$ at the current spa- 
cial point. Similarly, we define local solid volume fraction $\phi_{s}$. In two-phase (fluid and solid phases) porous media, we have obviously $\phi_{f}+\phi_{s}=1$. The nominal porosity measures the current fluid phase volume per unit reference total volume, denoted by $\Phi_{f}$. Since $\phi_{f} d \Omega_{t}=\Phi_{f} d \Omega_{0}$, which captures the current fluid volume, and $d \Omega_{t}=J d \Omega_{0}$ where $J=\operatorname{det}(F)$ is the Jacobian of deformation tensor $F$, we have the following relation:

$$
\Phi_{f}=J \phi_{f}
$$

\subsection{Swelling mechanism}

There are three energy sources governing the swelling dynamic of a polyacrylate gel: energy related to the mixing of the solvent and polymer, energy related to the ions osmosis and elastic energy of the polymer chains ${ }^{13}$. For the total free energy of the gel $\mathscr{F}$, we have:

$$
\mathscr{F}=\mathscr{F}_{\text {mix }}+\mathscr{F}_{\text {ion }}+\mathscr{F}_{\text {elastic }} .
$$

The first two types of energy $\left(\mathscr{F}_{\text {mix }}\right.$ and $\mathscr{F}_{\text {ion }}$ ) tend to stretch the polymer chains due to the uptake of solvent molecules, while the cross-linkers guarantee that the gel does not dissolve itself into the solvent and evokes the elastic energy ( $\mathscr{F}_{\text {elastic }}$ ) to balance out the stretching force. Swelling equilibrium is achieved when the total free energy reaches its minimum. Assuming the first two contributions to swelling are independent of each other, we have the total osmotic pressure $\Pi_{t o t}$ :

$$
\Pi_{t o t}=\Pi_{m i x}+\Pi_{i o n}
$$

where $\Pi_{m i x}$ denotes the osmotic pressure relates to mixing and $\Pi_{i o n}$ is the osmotic pressure related to ions osmosis. According to Horkay ${ }^{38}, \Pi_{i o n}$ is the dominant part of the two at a high swelling degree. In other words, Donnan theory (only) is adequate to describe the effect of the monovalent ions on the swelling of a polyacrylate hydrogel. The high swelling degree is the exactly the scenario that we are interested in, the total osmotic pressure in the current study is thus only the part related to ionic osmosis. Namely, $\Pi_{t o t}=\Pi_{i o n}$.

For a polyacrylate hydrogel (Fig. 1), Donnan osmosis leads to a process that results in the uptake of a large amount of solvent. Upon contact with the solvent (for example, pure water or physiological solutions), the positive sodium ions in the gel can relatively freely move around as the bond with the negative carboxylate ions is weakened due to the high dielectric constant of water. However, they cannot leave the gel since they are still weakly bonded to the negative carboxylate ions. As a result, the hydrogel acts like a semi-permeable membrane which allows the exchange of water molecules but not of sodium ions. Consequently, the osmotic pressure differences arise in and outside of the gel. The osmotic pressure due to ionic forces has to be compensated by the hydraulic pressure difference and that gives rise to the uptake of water.

We adopt Donnan theory to describe the osmotic contribution, ignoring interactions between ions. It is also assumed that the equilibrium of the ion phases is established instantly, in other words, at a much shorter time scale than the mass intake of the solvent. This assumption is justified by the following short

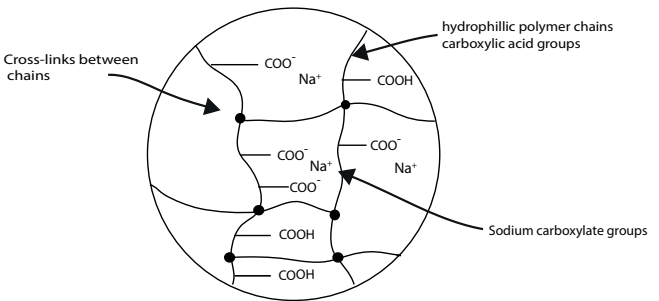

Fig. 1 Schematic illustration of polyacrylate gel structure

calculation. The diffusion coefficient of ions $d_{\text {ion }}$ is of the magnitude $10^{-9} \mathrm{~m}^{2} / \mathrm{s}^{39}$. The hydraulic pressure diffusion coefficient $d_{p}$ is estimated by the multiplication of hydraulic permeability $K$ and Young's modulus $E$. For a typical SAP gel, $K$ is of order $10^{-3} \mathrm{~mm}^{4} /(\mathrm{Ns})$ and $E$ is $10^{-2} \mathrm{~N} / \mathrm{mm}^{2}$. Therefore, we have $d_{i o n} / d_{p}=10^{2}$, which shows that the ion diffusion happens indeed much faster than mass fluid up-take for an SAP gel.

It is assumed that only uni-valent ions are present in the outer solution. Under the condition that the activity coefficient of the mobile ions is the same in the gel and in the equilibrium solution, Donnan equilibrium concentration for the ions inside the gel are derived ${ }^{19}$ :

$$
\begin{aligned}
& c^{+}=\frac{1}{2}\left(-c^{f c}+\sqrt{\left(c^{f c}\right)^{2}+4 \bar{c}^{2}}\right), \\
& c^{-}=\frac{1}{2}\left(c^{f c}+\sqrt{\left(c^{f c}\right)^{2}+4 \bar{c}^{2}}\right),
\end{aligned}
$$

where $c^{f c}$ denotes active fixed charge (carboxylate group) density and $\bar{c}$ is the external solution ions $\left(\mathrm{N}_{a}^{+}\right.$and $\left.\mathrm{Cl}^{-}\right)$molar concentration. As a result, the osmotic pressure inside and outside of the gel are given by van't Hoff law:

$$
\begin{aligned}
\pi_{\text {in }} & =R T \sqrt{\left(c^{f c}\right)^{2}+4 \bar{c}^{2}}, \\
\pi_{\text {out }} & =2 R T \bar{c} .
\end{aligned}
$$

The osmotic pressure related to the ions osmosis is thus derived as:

$$
\Pi_{i o n}=R T \sqrt{\left(c^{f c}\right)^{2}+4 \bar{c}^{2}}-2 R T \bar{c} .
$$

The chemical potential of the gel $\mu$, in this case, is composed of two parts: concentration related part (osmotic pressure) and the mechanical part (pore pressure). The pore pressure in the gel $p$ is thus defined as ${ }^{21}$ :

$$
p=\mu+\Pi_{i o n}
$$

To be able to describe the fluid-solid-ions coupled system quantitatively, governing equations supplemented with constitutive relations are needed. In our current swelling model, three equations govern the system. They are (linear) momentum balance equation, fluid content continuity equation and a Darcy type equation. Details of the governing equations are given in the three following subsections. 


\subsection{1 (Linear) momentum balance equation.}

Total stress $\sigma$ consists of Terzaghi effective stress $\sigma_{e f f}$ and pore pressure $p$ :

$$
\sigma=\sigma_{e f f}+p I
$$

This is a well-known relation in poromechanics ${ }^{40}$. The effective stress is derived from the elastic strain energy function for the gel $\mathscr{F}_{\text {elastic }}$ by the relation:

$$
\sigma_{e f f}=J^{-1} F \frac{\partial \mathscr{F}_{\text {elastic }}}{\partial C} F^{T}
$$

The strain energy function $\mathscr{F}_{\text {elastic }}$ takes the form ${ }^{41}$ :

$$
\mathscr{F}_{\text {elastic }}=\frac{1}{12} G \frac{\left(1+0.5 \phi_{s, 0} / J\right)}{\left(1-\phi_{s, 0} / J\right)} \ln ^{2}(\operatorname{det}(C))+\frac{1}{2} G(\operatorname{tr}(C))-3 \operatorname{det}(C)^{1 / 3},
$$

where $G$ is shear modulus and $\phi_{s, 0}$ is the initial solid volume fraction. This elastic strain energy represents the compressible NeoHooekan material law with the inclusion of the relation between Poisson's ratio $v$ and solid volume fraction $\phi_{s}: v=0.5 \phi_{s}=0.5 \frac{\phi_{s, 0}}{J}$. This relation suggests that the molecular network is incompressible when there is no fluid present $\left(\phi_{s}=1\right)$. As the swelling proceeds, the gel takes a smaller and smaller Poisson's ratio. This is a reasonable description of the mechanical property of the gel, since, as we can imagine, a swollen gel is much likely to experience volume change under compression due to fluid outflow than a dry molecular network. The linear momentum balance written in the Eulerian form is

$$
\nabla \cdot \sigma=0
$$

Its Lagrangian counterpart is

$$
\nabla_{X} \cdot T=0
$$

where the subscript $X$ denotes the divergence operator is taken with respect to the Lagrangian variable $X$; and $T$ is the first Piola-Kirchhoff stress tensor, related to the Cauchy stress tensor by $T=J \sigma F^{-T}$.

\subsubsection{Fluid content continuity equation.}

Let $V^{f}$ and $V^{s}$ denote the Eulerian velocity of the fluid and solid phase respectively. The Eulerian fluid continuity equation reads as:

$$
\frac{\partial \phi_{f}}{\partial t}+\nabla \cdot\left(\phi_{f} V^{f}\right)=0
$$

where we have made use of the assumption that the fluid is incompressible and therefore its true density is constant. If we rewrite this equation referring to the skeleton motion, we have:

$$
\frac{\partial^{s} \phi_{f}}{\partial t}+\phi_{f} \nabla \cdot V^{s}+\nabla \cdot q=0
$$

where the superscript "s" denotes the time derivative taken with respect to the solid skeleton and $q$ is defined as the filtration vector $q=\phi_{f}\left(V^{f}-V^{s}\right)$. After a "pull back" operation on the equation to the initial configuration, we arrive at the following form of the continuity equation:

$$
\frac{\partial \Phi_{f}}{\partial t}+\nabla_{X} \cdot Q=0
$$

where $Q$ is the Lagrangian vector attached to the original configuration and is related to its Eulerian counterpart as $Q=J F^{-1} q$. The nominal porosity $\Phi_{f}$ is given in equation (1). Using the fact that the solid phase is incompressible and there is no external mass source during swelling, we have the solid phase mass conservation:

$$
\phi_{s, 0}=J\left(1-\phi_{f}\right)=J-\Phi_{f} .
$$

Consequently, the fluid continuity equation reads:

$$
\frac{\partial J}{\partial t}+\nabla_{X} \cdot Q=0
$$

\subsubsection{Darcy type equation.}

The inflow of the fluid matter is driven by the gradient of chemical potential under isothermal conditions 42 ; while the equilibrium state is characterized by the overall uniform value for chemical potentials. A Darcy type equation relates the fluid flux to the chemical gradient. We have namely:

$$
Q=-K \nabla_{X} \mu
$$

where $K$ is related to the intrinsic (Eulerian) permeability $k$ and viscosity $\eta$ by $K=J F^{-1} F^{-T} k / \eta$. The $J F^{-1}$ part converts the Eulerian flux to the nominal one, and $F^{-T}$ converts the Eulerian gradient to the Lagrangian one. As to the specific form of permeability $k$ for an SAP gel, more discussion will follow in the next section. The system is further closed by the constitutive relation regarding $c^{f c 41}$ :

$$
c^{f c}=c_{0}^{f c} \frac{\phi_{0, f}}{J-\phi_{0, s}},
$$

where $c_{0}^{f c}$ and $\phi_{0, f}$ denotes the initial fixed charge density and initial porosity respectively.

\subsection{Strain-dependent permeability}

Generally, hydraulic permeability changes according to the pore structure of the porous material. Although such change is neglected in linear poroelasticity, where the deformation is assumed to be infinitesimal, such change plays an significant role in studying the dynamic process involving (extremely) large deformations. In the case of SAP hydrogels, as the outer solution starts to flow into the gel, the size of the pore is enlarged. However, as the mass fluid permeation takes time, the inner core of the gel stays with small pore size. Thus, heterogeneity in hydraulic permeability inside a transient swelling gel arises.

It is natural to assume the permeability to be a function of the porosity $\phi_{f}$. Making use of the fact that the volume of the solid part stays unchanged during swelling, the porosity $\phi_{f}$ at the different position of the gel can be directly calculated from the volume ratio $J$ using the relation:

$$
\phi_{f}=1-\frac{\phi_{s, 0}}{J}
$$


There are different porosity-permeability relationships available designed for specific pore structures. Kozeny-Carman model $^{43,44}$ is one of the most widely known and simplest models describing such a relationship. It was originally derived from the experimental measurement of beds of closely packed spheres. A nonlinear relationship between the volumetric strain and permeability is observed by means of an experiment on articular cartilage ${ }^{45}$. Under the condition of molecular incompressibility, the relationship between permeability and porosity is derived ${ }^{46}$ as:

$$
k\left(\phi_{f}\right)=k_{0}\left(\frac{1-\phi_{f, 0}}{1-\phi_{f}}\right)^{M},
$$

where $k_{0}$ is initial intrinsic hydraulic permeability and $M$ is a parameter (a positive number) that can be fitted for specific material. This relation is frequently used in biomechanical studies $^{47}$. Extensive experiments have been done regarding polyacrylamide gel over the years. The dependence of friction coefficient of a polyacrylamide gel on temperature, the concentration of the polymer solution and the concentration of the crosslinker was reported by Tokita ${ }^{48}$. Later, further investigation into the influence of flow velocity and polymer concentration on the gel's permeability using different experimental techniques was done by Grattoni ${ }^{49}$. Recently, Engelsberg ${ }^{50}$ related the friction coefficient to permeability and proposed the following law:

$$
k\left(\phi_{f}\right)=k_{0} \frac{\phi_{f}}{\left(1-\phi_{f}\right)^{\beta}},
$$

where the $\beta$ is a material parameter and has been suggested the value of $1.5,1.85$ and $1.75^{48-50}$. The normalized version of (24) is:

$$
k\left(\phi_{f}\right)=k_{0} \frac{\left(1-\phi_{f, 0}\right)^{\beta}}{\phi_{f, 0}} \frac{\phi_{f}}{\left(1-\phi_{f}\right)^{\beta}} .
$$

\section{Numerical simulations}

The numerical implementation of MHFEM features a three-field mixed formulation $(x, Q, \mu)$. The motivation for choosing such a formulation lies in the fact that the local mass conservation is naturally preserved. Unlike in standard FEM (Fig. 2), the mixed formulation avoids numerical differentiation by calculating flux as an independent variable. The advantages of local mass conservation property become obvious when the permeability tensor is non-homogeneous ${ }^{22,23}$. This observation gives rise to the use of the mixed formulation in this study, considering the fact that the permeability tensor is usually not uniform over the domain during swelling. Moreover, an accurate calculation of the (transient) flux field has a direct influence on the accuracy of the (transient) deformation field.

On the other hand, from the computational point of view, the computational cost is not necessarily increased due to the addition of flux field. Hybridization procedure and static condensation technique ${ }^{51}$ guarantee that the eventual system can be reduced to two fields again: $x$ and $\lambda$, where $\lambda$ is the Lagrange multiplier defined on each edge with the physical meaning of chemical potential. For more details see (how to cite my first paper). As a result, the tangent stiffness matrix is not significantly larger than the one with $(x, \mu)$ formulation.

In this section, the proposed numerical model is first verified with two classic problems in poroelasticity in a three-dimensional setting. Next, we introduce a MATLAB-calculated solution (for free swelling) in one dimension as a benchmark for the calculations in finite deformation regime. Having observed a perfect match between simulation solutions and analytical solutions, we proceed with three representative numerical examples to demonstrate the capability of the proposed numerical model.

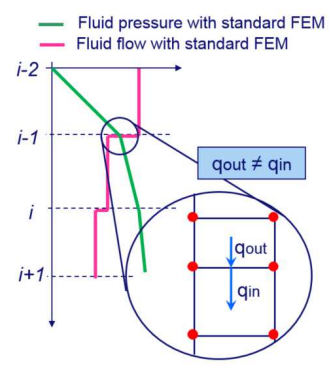

Fig. 2 A schematic illustration of violation of local mass conservation. There is loss of mass across the element boundary due to numerical differentiation.

\subsection{Solution verification: Terzaghi's problem and Cryer's problem}

We first carry out simulations of the Terzaghi's problem ${ }^{52,53}$ in three-dimensional settings. Specifically, we compress the sample in the z-direction (0.01 MPa) and disallow any displacement in the $\mathrm{x}$ - and $\mathrm{y}$-directions. Fluid drainage happens at the upper surface and the lower surface is impermeable. Note that in Terzaghi's problem there are no fixed charge groups involved. Therefore, the chemical potential is reduced to fluid pressure. Moreover, the analytical solution is limited to small deformation. We increase the shear modulus 200 times (30 MPa) to make sure that the deformation is infinitesimal. Fig. 3 plots the simulation and analytical solutions.

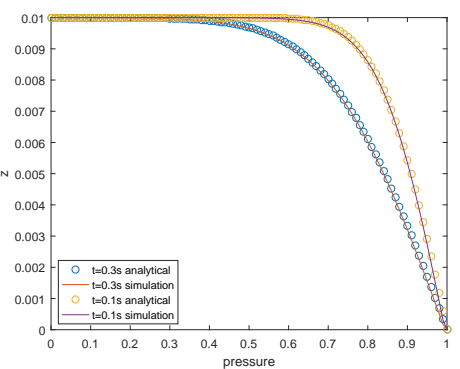

Fig. 3 Analytical and simulation solutions of the pressure profile along the $\mathrm{z}$-direction at time $\mathrm{t}=0.3 \mathrm{~s}$ and $\mathrm{t}=0.1 \mathrm{~s}$

The next classic problem is the Cryer's problem ${ }^{54}$. In Cryer's problem, a spherical soil sample is subjected to a constant pressure $(0.1 \mathrm{MPa})$ from all sides. Cryer's original solution ${ }^{54}$ calculated the pressure at the middle of the sphere. In Fig. 4 the simulation solution is compared to the analytical one. The overshoot of the pressure at the beginning stage in Cryer's problem is the 
well-known Mandel-Cryer effect. We observe that good matches between the analytical and simulation solutions are achieved for both Terzeghi's and Cryer's problems.

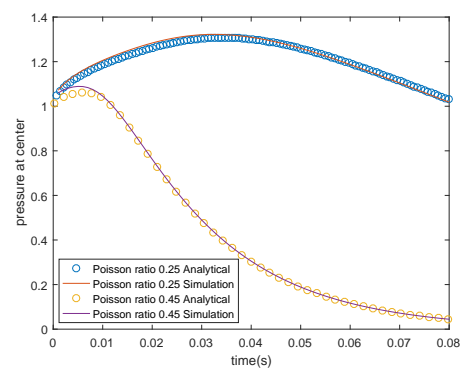

Fig. 4 Analytical and simulation solutions of the time evolution of the pressure at the center

\subsection{Solution verification in finite deformation regime}

Having compared the solutions to the classic problems in poroelasticity, we next present a MATLAB-calculated solution in finite deformation regime in one dimension. The governing equations in one dimension can be reduced to one parabolic type of equation of volume ratio $J$ :

$$
\frac{\partial J}{\partial t}-\frac{\partial}{\partial t}\left(\frac{k}{J} \frac{\partial\left(\sigma_{e f f}-\pi\right)}{\partial x}\right)=0,
$$

where $k, \sigma_{\text {eff }}$ and $\pi$ are nonlinear functions of $J$, representing the hydraulic permeability, effective stress and osmotic pressure respectively. Initial and boundary conditions for free swelling are:

$$
\begin{aligned}
J(x, 0) & =1, \quad 0 \leq x \leq 1 \\
-\frac{k}{J} \frac{\partial\left(\sigma_{e f f}-\pi\right)(J(1, t))}{\partial x} & =0, \quad 0 \leq t \leq T \\
\left(\sigma_{e f f}-\pi\right)(J(0, t)) & =-2 R T \bar{c}, \quad 0 \leq t \leq T .
\end{aligned}
$$

Such a partial differential equation can be solved by MATLAB internal solver and solution is used as a benchmark. We compare both the deformation and chemical potential field of the MHFEM solution to the benchmark solution in Fig. 5 and 6. The good agreement between MHFEM solution and the benchmark solution gives us confidence in terms of the reliability of the MHFEM model.

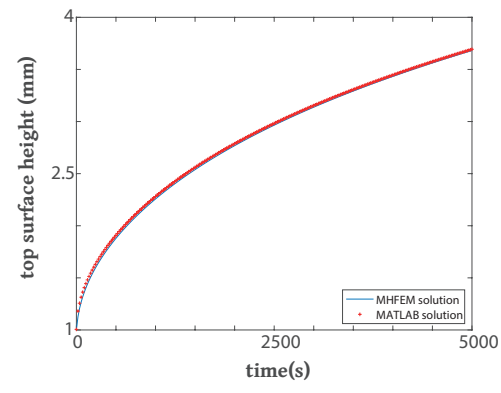

Fig. 5 Top surface trajectories compare

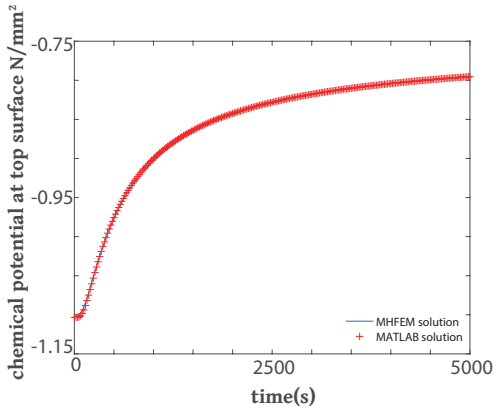

Fig. 6 Chemical potentials at the top surface compare

\subsection{Numerical examples}

The capability of the model is demonstrated by three numerical examples. We focus on two academically and industrially relevant scenarios: free swelling of a gel and squeezing fluid out of a swollen gel. The geometry of the gel is taken to be three dimensional, specifically, a cube, a sphere or a random irregular shape (derived by means of scanning a real-life SAP particle). The set of material parameters used in the model is given in Table 1. Equation (25) is chosen for the strain-dependent permeability relationship with $\beta=1.5$.

Table 1 Model parameters

\begin{tabular}{lll}
\hline Parameters & value & unit \\
\hline Shear modulus G & 0.015 & $\mathrm{~N} / \mathrm{mm}^{2}$ \\
Initial hydraulic permeability $k_{0}$ & $10^{-3}$ & $\mathrm{~mm}^{4} /(\mathrm{Ns})$ \\
Initial porosity $\phi_{f, 0}$ & 0.83 & $\mathrm{~K}$ \\
Universal gas constant R & 293 & \\
Osmotic coefficient $\Gamma$ & 1 & $\mathrm{~mol} / \mathrm{ml}$ \\
Initial fixed charge density $c_{0}^{f c}$ & $3.32 \times 10^{-4}$ & \\
\hline
\end{tabular}

\subsubsection{Free swelling of a spherical hydrogel}

Our first numerical example is the free swelling of a spherical gel partitioned into eight quadrants (Fig. 7). The initial radius $r_{0}$ equals $0.25 \mathrm{~mm}$ and is discretized by 2560 linear hexahedral elements. Outer solution concentration $(\bar{c})$ is taken to be $1.54 \times$ $10^{-5} \mathrm{~mol} / \mathrm{ml}$. The swelling ratio at the equilibrium state is about 70.7 .

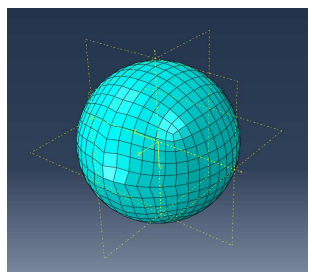

Fig. 7 Spherical gel geometry

The boundary conditions are prescribed as follows: $\mu=-2 R T \bar{c}$ for all elements on the outer surface; Origin O (in all three dimensions) and one cutting plane of one of the quadrants (in its initial 2D plane) are fixed to avoid rigid motions. Initial conditions are: $\mu=-R T \Gamma \sqrt{\left(c_{0}^{f c}\right)^{2}+4 \bar{c}^{2}}$ and $x=X_{0}$. We introduce dimensionless quantities to present the simulation results. By means of scaling, 
normalized quantities are derived:

$$
\tilde{t}=\frac{t}{t_{c}}, \quad \tilde{\sigma}=\frac{\sigma}{\sigma_{c}}, \quad \tilde{\mu}=\frac{\mu}{\sigma_{c}}, \quad \tilde{k}=\frac{k}{k_{c}},
$$

where the scaling factors for time, stress, chemical potential and permeability are $t_{c}=r_{0}^{2} / 2 R T \bar{c} k_{0}, \sigma_{c}=2 R T \bar{c}$ and $k_{c}=k_{0}$.

The location, magnitude and direction of extremal stresses (maximal and minimal principal and shear stress) are useful to be identified in structure design. Above a certain limit of these stresses, material failure might occur (e.g. the initiation of fracture). In Fig. 8, the distribution of normalized maximal principal stress at the surface at four different transient swelling moments was plotted. We plotted the extremal stress distribution on the outer surface (not in the depth direction) since the outer surface swelled first and experienced the largest local deformation and thus represented the whole range of extremal stresses. The evolution of maximal principal and shear distribution on the surface in time can be found in the Electronic Supplementary Information (Movie 1 and Movie 2). Fig.8 shows that the maximal principal stresses tend to be uniform over the sphere surface as the swelling continues. The distribution of the stresses in transient states exhibits quadrant-wise symmetry. The authors speculate the loss of uniformity over the surface is related to physical surface instabilities. Moreover, Fig.8(b) indicates that the spherical shape was temporarily replaced by a shape close to cube. Later, the maximal principal stress evolved into a hexahedral spherical harmonic buckling mode (Fig. 8(c)).

Fig. 9 and 10 present the normalized chemical potential and permeability in the depth direction during transient swelling. The complete chemical potential evolution movie is included in ESI (Movie 3). The transient states exhibited strong inhomogeneity in terms of chemical potential and permeability. For both chemical potential and permeability, the outer surface has the largest value gradually decreases along the radius to the inner core. However, one exception is shown in Fig. 9(a). Given a closer look at the chemical distribution at the very beginning of the swelling, the lowest chemical potential appears not in the core but the layer underneath the top surface. An intuitive explanation is that at the moment right after the sample starts to swell, the inner core is dry and undeformed. As a result, the pressure related to the expansion of the outer surface is thus applied to the neighboring layer which leads to the lowest chemical potential of the whole sample. This phenomenon is similar to the well-known MandelCryer effect in poroelasticity, which described the non-monotonic response of pressure to external loading ${ }^{55}$.

\subsubsection{Free swelling of a realistic SAP particle}

The SAP particles were measured in a micro-CT setup with $6 \mu \mathrm{m}$ resolution. They were put on a Petri disk such that they did not touch any other particles. Then they were imaged to obtain a stack of 2-dimensional images. Then they were imported into avizo image analyzing software, where the surface plots were extracted. We make use of the commercial FEM simulation software ABAQUS to recover their volumetric geometries and generate meshes on them. Due to the irregularity in the geometry, automatic hexahedral element generation is no longer possible. A natural alternative is the 10-node quadratic tetrahedral element, which is reported to have equivalent performance as the linear hexahedral element ${ }^{56}$. One example of the (meshed) particle geometries is given in Fig. 11. Although there is no longer symmetry preserved in such a geometry, we divide the gel domain into eight (approximately equal-sized) quadrants. As the first attempt, we simulate a quadrant of such a complex geometry (Fig. 12) using 365 quadratic tetrahedral elements.

The outer three-dimensional and irregular surface (where node A,B,C are in) is in contact with the outer solution whose concentration $(\bar{c})$ equals $1.54 \times 10^{-4} \mathrm{~mol} / \mathrm{ml}$. The swelling ratio in the equilibrium state is about 12.34. The outer (irregular) surface assumes Dirichlet boundary condition $\left(\Gamma_{D}^{\mu}\right)$ for chemical potential $(\mu=-2 R T \bar{c})$. The inner surfaces (OAB,OBC,OAC) takes no flow boundary conditions $\left(\Gamma_{N}^{\mu}\right)$. The symmetry requirement for the position field asks for no displacement in $z$ direction for surface $\mathrm{OAB}$, no displacement in $x$ direction for surface OAC and no displacement in $y$ direction for surface OCB. At the end, the whole sample has traction free boundary conditions. To sum up, we have:

$$
\left\{\begin{array}{r}
x(X, t)=X_{0} \text { on } \Gamma_{D}^{u} \times[0, T], \\
T(X, t) \cdot n=0 \text { on } \Gamma_{N}^{u} \times[0, T],
\end{array}\right.
$$

and

$$
\left\{\begin{aligned}
\mu(X, t)= & -2 R T \bar{c} \text { on } \Gamma_{D}^{\mu} \times[0, T], \\
Q(X, t) \cdot n & =0 \text { on } \Gamma_{N}^{\mu} \times[0, T],
\end{aligned}\right.
$$

The initial conditions are:

$$
\begin{aligned}
x(X, 0) & =X_{0}, \\
\mu(X, 0) & =-R T \Gamma \sqrt{\left(c_{0}^{f c}\right)^{2}+4 \bar{c}^{2}} .
\end{aligned}
$$

The maximal principal stresses distribution at four different moments are given in Fig. 13. The complete evolution of maximum principal and shear stresses and chemical potential of the quadrant during swelling are included in ESI (Movie 4,5 and 6). Just like we observed in the spherical simulations, the extremal stresses tend to distributed uniformly as the swelling degree increases. However, due to the complexity of the geometry, the distribution of the extremal stresses becomes much more tricky to predict. The area near point $\mathrm{C}$ and the middle region of the outer surface seem to be the area of interest with the most often appearance of the maximum/minimum value of the extremal stresses. The distribution of chemical potential values are alike the spherical cases with higher values at the outer surface and gradually decrease along the radius to the core.

\subsubsection{Squeezing a cubic gel}

In this example, we let a cubic gel discretized by $6 \times 6 \times 6$ linear hexahedral elements subject to compressive surface forces in all three principal directions to simulate squeezing the solvent out of the gel mechanically. The gel was initially in a stress free-state and in equilibrium with the outer solution with the concentration $\tilde{c}=0.037 \mathrm{~mol} / \mathrm{ml}$. All faces of the cube are in fluid exchange with the outer solution and are subjected to the inward normal compressive surface forces. Specifically, the boundary conditions can 


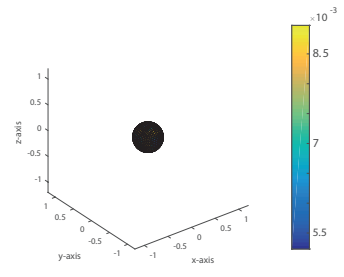

(a) $\tilde{t}=0$

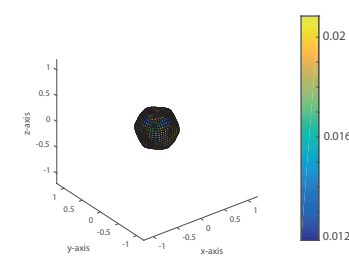

(b) $\tilde{t}=0.003$

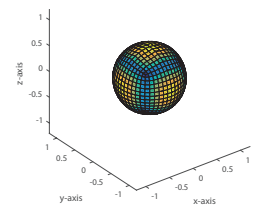

(c) $\tilde{t}=0.03$
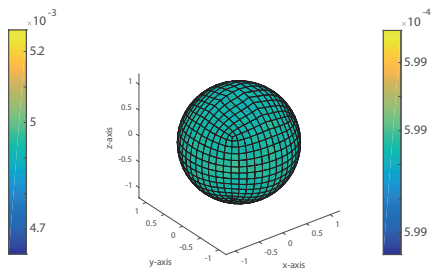

(d) $\tilde{t} \rightarrow \infty$

Fig. 8 Maximal principal stress on the gel sphere at different moments

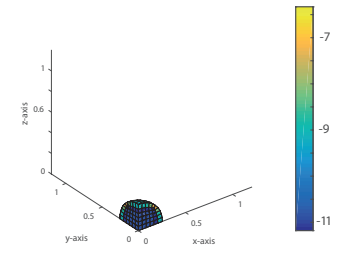

(a) $\tilde{t}=0$ (b) $\tilde{t}=0.003$

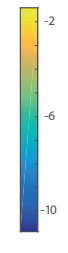

(c) $\tilde{t}=0.03$
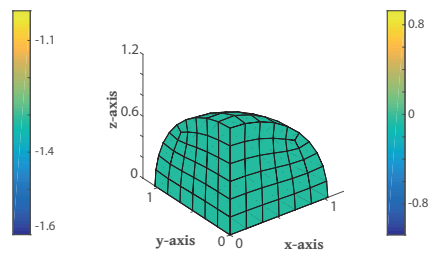

(d) $\tilde{t} \rightarrow \infty$

Fig. 9 Normalized chemical potential in the gel sphere at different moments

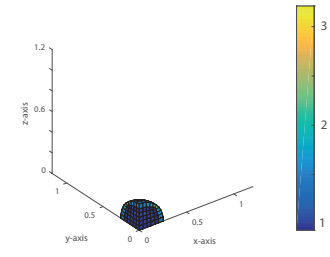

(a) $\tilde{t}=0$

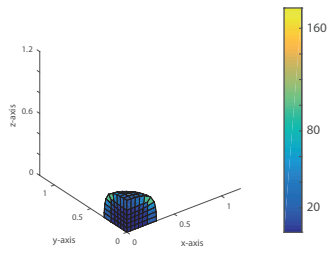

(b) $\tilde{t}=0.003$

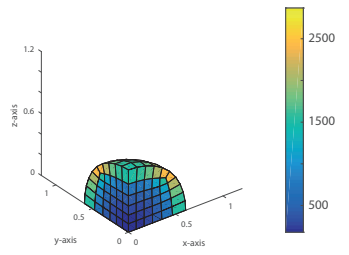

(c) $\tilde{t}=0.03$

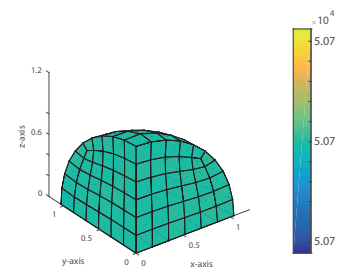

(d) $\tilde{t} \rightarrow \infty$

Fig. 10 Normalized permeability in the gel sphere at different moments

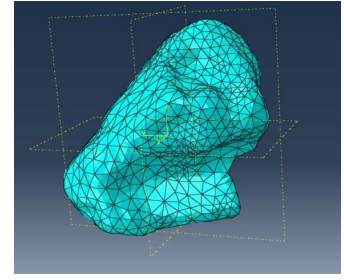

Fig. 11 Tetrahedral mesh on a SAP particle

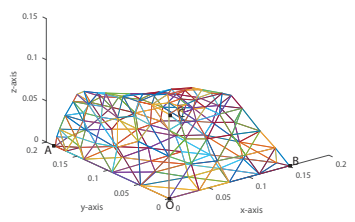

Fig. 12 Tetrahedral mesh on a quadrant of a SAP particle

be written as:

$$
\left\{\begin{aligned}
x(X, t) & =X_{0} \text { on } \Gamma_{D}^{u} \times[0, T] \\
T(X, t) \cdot n & =F_{e x t} \text { on } \Gamma_{N}^{u} \times[0, T]
\end{aligned}\right.
$$

and

$$
\left\{\begin{array}{rll}
\mu(X, t) & =0 & \text { on } \Gamma_{D}^{\mu} \times[0, T], \\
Q(X, t) \cdot n & =0 & \text { on } \Gamma_{N}^{\mu} \times[0, T],
\end{array}\right.
$$

where $\left\|F_{\text {ext }}\right\|=0.03 \mathrm{~N} / \mathrm{mm}^{2}$ denotes the magnitude of the compressive surface force. The initial conditions are:

$$
\begin{aligned}
& x(X, 0)=X_{0}, \\
& \mu(X, 0)=\left\|F_{\text {ext }}\right\| .
\end{aligned}
$$

The chemical potential and the geometry change of the cubic gel in the initial, transient and equilibrium states are given in Fig. 14. A complete time evolution of the chemical potential contour plot is given in ESI (Movie 7). At the initial state, the chemical potential is uniform and equals the applied force (Fig.14(a)). Under squeezing, the outer most corner undergoes the largest deformations since it suffered from the most compressive forces (from all three directions). However, as it took time for the solvent to flow out of the gel, the compressive force also led to temporary bulging of the cubic gel on each face as indicated in Fig. 14(b) and Fig. 14(c). Eventually, as the solvent got completely depleted (chemical potential equals zero uniformly), the compressive forces were completely applied on the solid skeleton and the cubic shape was recovered as shown in Fig. 14(d). 


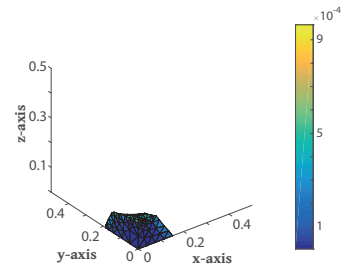

(a) $t=0.01 \mathrm{~s}$

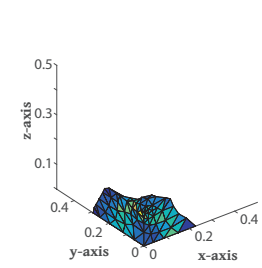

(b) $\mathrm{t}=2.61 \mathrm{~s}$

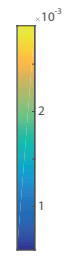

(c) $t=19.3 \mathrm{~s}$

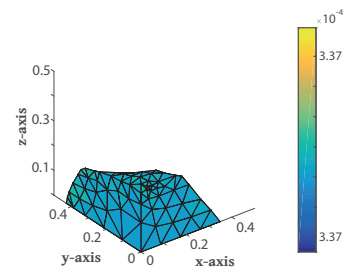

(d) $t=10000 \mathrm{~s}$

Fig. 13 Maximal principal stress on the gel sphere at different moments

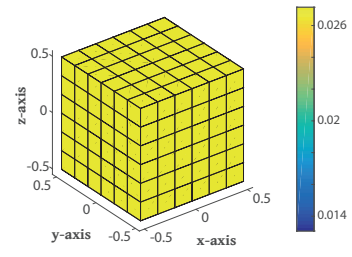

(a) $t=0.005 \mathrm{~s}$

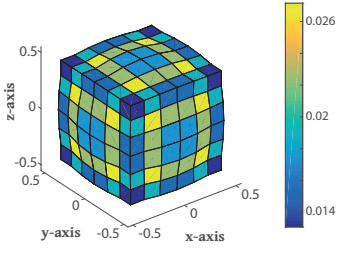

(b) $\mathrm{t}=124.8 \mathrm{~s}$

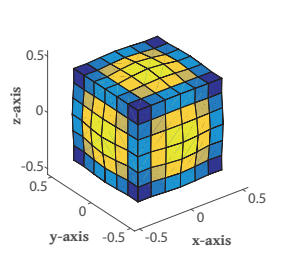

(c) $t=763.3 \mathrm{~s}$
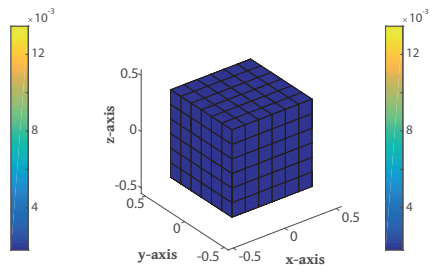

(d) $t=150000 \mathrm{~s}$

Fig. 14 Chemical potential in a cubic gel at initial, during squeezing and equilibrium states

\section{Surface instability study}

In this section, a linear perturbation analysis following the work presented in ${ }^{30}$ but using the proposed material law is presented. Next, we make use of the MHFEM swelling model to simulate a thin gel slab swelling fixed on a rigid substrate in two dimensions. Surface instability (typically wrinkling) is observed. Observations related to the onset of the instability are summarized. At last, a three-dimensional swelling simulation of a tubular structure with constraints is presented. The circumferential wrinkling behavior is qualitatively compared with experimental results ${ }^{57}$. In this study, we aim to demonstrate the capability of the proposed numerical model in simulating complex swelling-induced surface instabilities as well as bridge the knowledge we obtained from both sources (theoretical analysis and simulations) to gain a better understanding about the onset of the buckling instability.

\subsection{Linear perturbation analysis}

In perturbation analysis, we perturb a swollen state thus it is essentially a quasi-static analysis. Also, only linear contributions of the infinitesimal perturbation are considered while the equilibrium swollen state is computed from the non-linear theory. To examine the stability of the swelling deformation, we perturb the swollen state with small perturbations in both $\mathrm{x}$ and $\mathrm{y}$ directions, $u_{1}$ and $u_{2}$. The swelling is laterally confined (in the x-direction), thus swelling is only allowed in the y-direction. Therefore, the swollen state can be characterized by the ratio of the gel thickness in y-direction $\lambda$.

The deformation tensor $F$ in the perturbed state is given by:

$$
\widetilde{F}=\left(\begin{array}{cc}
1+\frac{\partial u_{1}}{\partial x} & \lambda \frac{\partial u_{1}}{\partial y} \\
\frac{\partial u_{2}}{\partial x} & \lambda\left(1+\frac{\partial u_{2}}{\partial y}\right)
\end{array}\right) .
$$

Thus, the volume ratio $J$ is calculated as

$$
J=\operatorname{det}(\widetilde{F}) \approx \lambda(1+\varepsilon)
$$

where $\varepsilon=\frac{\partial u_{1}}{\partial x}+\frac{\partial u_{2}}{\partial y}$. Demanding the linear momentum balance to hold at the perturbed state, we have:

$$
\widetilde{T}=0
$$

where $\widetilde{T}$ is the first Piola-Kirchoff stress in the perturbed state. Note that for the perturbed stress tensor, only the effective part is modified based on the perturbation. The hydraulic pressure part $p$ stays unchanged and satisfies the equilibrium condition at the unperturbed state:

$$
\sigma_{e f f}-p I=0 .
$$

Using Fourier transform to solve equation (41), following the derivation presented in ${ }^{30}$, one can derive critical swelling ratio $\lambda_{c r}$ by solving the following nonlinear equation:

$$
\operatorname{det}\left(\begin{array}{cccc}
1 & 1 & 1 & 1 \\
-\lambda & \lambda & -\beta & \beta \\
\left(\lambda+\frac{1}{\lambda}\right) e^{k h_{0}} & -\left(\lambda+\frac{1}{\lambda}\right) e^{-k h_{0}} & 2 \beta e^{k \beta h} & -2 \beta e^{-k \beta h} \\
2 \lambda e^{k h_{0}} & 2 \lambda e^{-k h_{0}} & \left(\lambda+\frac{1}{\lambda}\right) e^{k \beta h} & \left(\lambda+\frac{1}{\lambda}\right) e^{-k \beta h}
\end{array}\right)=0
$$


where $k$ is the wave number, $h_{0}$ is the initial thickness, $h=\lambda h_{0}$ is the thickness at the unperturbed state. At last,

$$
\beta=\sqrt{\left(1+\lambda g_{\varepsilon}(\lambda)\right) /\left(\lambda^{2}+\lambda g_{\varepsilon}(\lambda)\right)},
$$

where $g_{\varepsilon}(\lambda)$ is nonlinear function of $\lambda$ derived from the specific constitutive relations. Using the given constitutive relations (equation 12 ), $g_{\varepsilon}$ is given by:

$$
g_{\varepsilon}(\lambda)=-\frac{1}{6} \frac{\ln \lambda}{\lambda} h_{\varepsilon}(\lambda)-\frac{1}{6}\left(\frac{1}{\lambda^{2}}-\frac{\ln \lambda}{\lambda^{2}}\right) h(\lambda)+\frac{1}{3} \lambda^{-4 / 3},
$$

where

$$
\begin{aligned}
h(\lambda) & =-1+3 \frac{-\lambda^{2}+\phi_{s, 0}^{2}+\ln (\lambda) \lambda \phi_{s, 0}}{\left(-\lambda+\phi_{s, 0}\right)^{2}}, \\
h_{\varepsilon}(\lambda) & =3 \frac{\phi_{s, 0} \lambda \ln \lambda+\phi_{s, 0}^{2} \ln \lambda+3 \phi_{s, 0}^{2}-3 \phi_{s, 0} \lambda}{\left(-\lambda+\phi_{s, 0}\right)^{3}} .
\end{aligned}
$$

In both analysis and simulation, we set the initial thickness $h_{0}$ to be $0.5 \mathrm{~mm}$ and initial length. In Fig. 15 the critical swelling ratios as function of wave number $k h_{0}$ with different initial porosity $\phi_{f, 0}$ are plotted. Just as derived from ${ }^{30}$, the critical swelling ratios decrease initially as the wave number increases but stop decreasing when the wave number exceeds a certain limit. In other words, the perturbations with longer wavelength tend to stabilize the surface, whereas there exists a limit for the shorter wavelength end beyond which the critical swelling ratio stays the same. On the other hand, as the initial porosity $\phi_{f, 0}$ decreases, which correspond to a smaller degree of swelling, the predicted critical swelling also decreases.

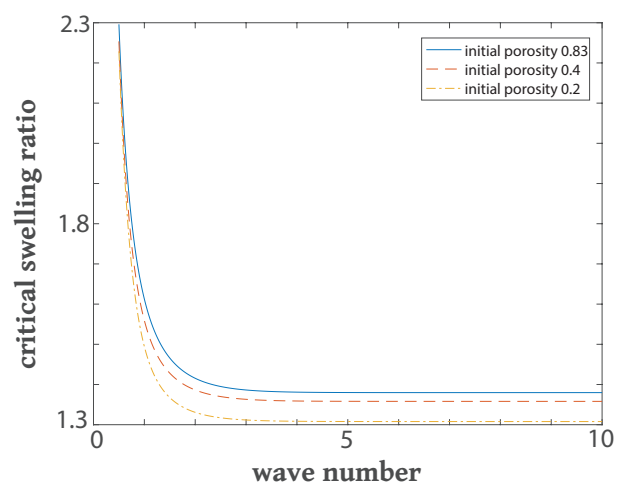

Fig. 15 Critical swelling ratio curves under different initial porosity

\subsection{Swelling of a thin gel slab on a rigid substrate in 2D}

Swelling under certain constraint induces a compressive stress in the gel. Such compressive stresses are observed in experiments $21,28,32,33$ and identified as the source of surface instabilities (wrinkling and creasing). Theoretical work based on energy analysis ${ }^{58}$ and linear perturbation ${ }^{30}$ predicts the critical swelling ratio at the equilibrium state. However, surface instabilities also happen during transient swelling. In this section, we focus on a $2 \mathrm{D}$ simulation of a thin gel slab attached to a rigid surface using the proposed swelling model. Since the gel slab is fixed in the plane and its dimension in the plane is significantly larger than in out-of-plane direction, the swelling will only happen in the thickness direction.

The schematic illustration of the initial geometry is given in Fig.16. The initial thickness (distance between $A B$ ) is set to be 0.5 $\mathrm{mm}$. The bottom side of gel slab is fixed to the rigid surface $\mathrm{BC}$ and with zero flux. The top side (AD, length $5 \mathrm{~mm}$ ) is in touch with the outer solution (with ion concentration $2.1 \times 10^{-5} \mathrm{~mol} / \mathrm{ml}$ ) and traction free. On the two sides (AB and $C D$ ) symmetric boundary conditions apply: zero displacements, shear and flux in the $\mathrm{x}$ direction.

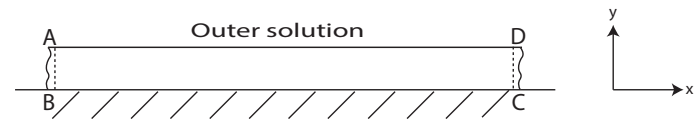

Fig. 16 Schematic illustration of a thin gel layer attached to a rigid surface

Using above simulation set-up, surface instabilities were observed during transient swelling at non-equilibrium state without perturbations. Typically, the surface instabilities initially presented itself as smooth wave-like wrinkles. As the swelling went on, the smooth waves developed into crease-like wrinkles with deep grooves (see Fig. 17). However, since there was no contact defined in the model, post-buckling behavior (creasing typically) was not able to be captured by the current numerical model. The negative values of normal stress in Fig. 17 again verifies the compressive stress rose during transient swelling.
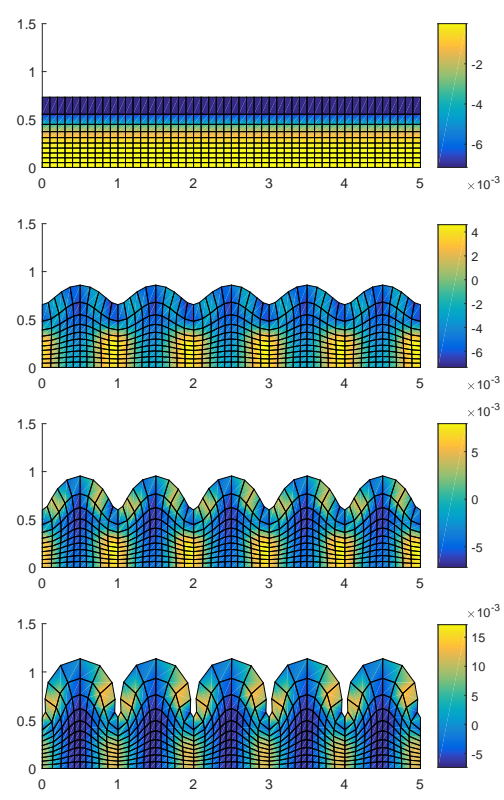

Fig. 17 Wrinkle development at the top surface of a swelling hydrogel slab on a rigid surface with color contour for in-plane normal effective stress $\sigma_{11}$

Simulation-wise, the onset of surface instability is usually iden- 


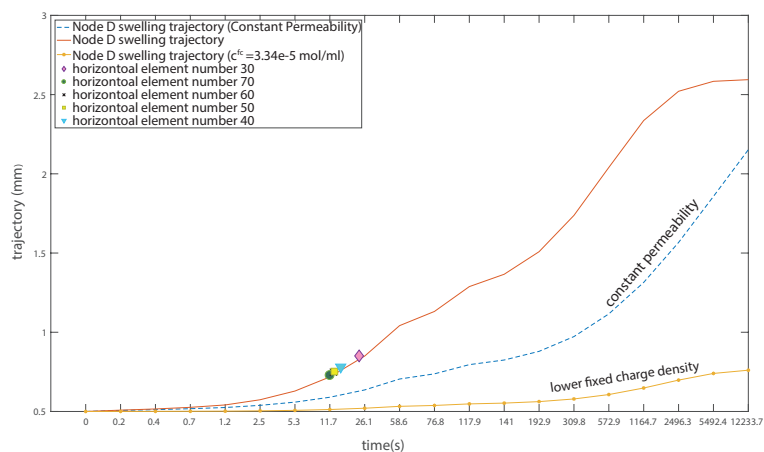

Fig. 18 Trajectories of node $D$ during swelling with different mesh sizes and swelling conditions

tified by a sharp turn (discontinuity in differential) in the trajectories. In Fig. 18, the trajectories of node D is plotted for variable permeability, constant permeability and with lower fixed charge density. For the variable permeability, as we gradually increased the number of horizontal elements, the onset of surface instability appeared. Specifically, the more horizontal elements, the sooner the wrinkles appeared. For constant permeability (which implies a less sharp change in displacement) and lower initial fixed charge density (which implies smaller swelling ratio in the equilibrium state), there were no surface instabilities observed throughout the swelling even for a large number of horizontal elements. We note that as the number of elements increases, the simulated critical swelling ratio starts to approach the theoretical prediction of the critical swelling ratio (about 1.4, see Fig. 15). This finding is in agreement with the work of Bouklas et al. ${ }^{59}$, where the authors also found that the instability delays depending on the element size. We speculate that the finite dimensional approximation of the geometry acts as a "low-pass filter", by use of which the smallest deformations caused by surface instabilities are filtered out.

In spite of mesh-dependency and delay in the prediction of the onset of surface instabilities using given numerical approach, simulations results can still provide some useful insights into the influence of various swelling-related factors on the onset of instability. In what follows, we investigate the influence of material parameters (shear modulus, initial fixed charge density and initial porosity) and the choice of permeability laws on the onset of instabilities using a fixed mesh size and time step size. We also relate our simulation results with experimental results reported different literature.

First of all, we investigate the influence of choice of permeability laws on the onset of surface instabilities. Permeability laws typically influence the speed of swelling but not the final swelling degree. A faster swelling process should result in more local compressive stress during transient swelling. This conclusion is supported by the simulation results. Using exactly the same material parameters and boundary conditions, only varying the choice of permeability laws, simulations are carried out for different initial fixed charge densities $\left( \pm 50 \%\right.$ of the standard value $3.32 \times 10^{-4}$ $\mathrm{mol} / \mathrm{ml}$ ). Fig. 19 shows that simulations with variable permeabil- ity laws exhibited surface instabilities during transient swelling; while with constant permeability (which means slower volume increase, see also Fig. 18) there appeared no surface instabilities throughout the swelling process and reached an equilibrium state with a final swelling ratio ranging from 3.5 to 6.5 . Note that permeability law 1 refers to the permeability law given in equation (23) with $M=2$ and law 2 refers to equation (25) with $\beta=2.5$. Although the form of (variable) permeability laws are different, the swelling ratios at the onset of instabilities were very close to each other for all the initial fixed charge densities.

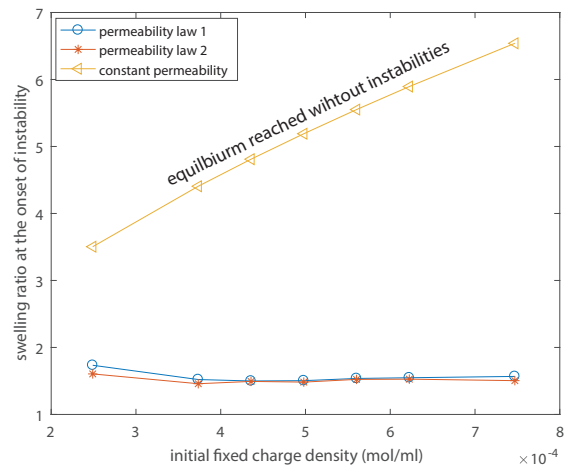

Fig. 19 Critical swelling ratios with different permeability laws (permeability law 1, permeability law 2 and constant permeability) under given initial fixed charge density range.

Next, we investigate the influence of shear modulus and initial porosity on the onset of instabilities. A larger shear modulus suggests a stiffer gel (usually achieved by higher cross-linking degree) and therefore leads to a smaller swelling degree in the equilibrium state. On the other hand, a smaller initial porosity suggests a smaller degree of swelling in equilibrium. The double $y$-axis plot (Fig.20) shows that there is no strict monotonic correlation between these two parameters and the swelling ratio at the onset of instabilities. As a matter of fact, the swelling ratios fall pretty much into a range between 1.45 and 1.65 for shear modulus between $6 \mathrm{kPa}$ and $24 \mathrm{kPa}$ and initial porosity between 0.6 and 1. The swelling ratio at the onset of instability (or equivalently critical strain) we found here is lower than the value reported in other literature. Specifically, Trujillo et al. ${ }^{32}$ and Drummond et al. ${ }^{60}$ reported the experimental results of 2 and 2.6 for creasing. Hong et al. ${ }^{58}$ derived the ratio to be 2.4 analytically using energy method. The discrepancy can be caused by the difference between "creasing" and "wrinkles" 26,33. The critical conditions reported in the literature are applicable for creasing; while the critical swelling ratio we recorded is the first moment of irregularity in displacement, in other words, the beginning of wrinkles.

At last, the boundary between stable and unstable states is studied. By running simulations using given shear modulus and initial fixed charge density pairs, the stable/unstable states were recorded. As shown in Fig. 21, towards the higher shear modulus and lower fixed charge density end, the gel slab is most likely to be stable (indicated by red dots) and the other end (lower shear modulus and higher initial fixed charge density) to be unstable (indicated by blue diamond markers). These results are in 


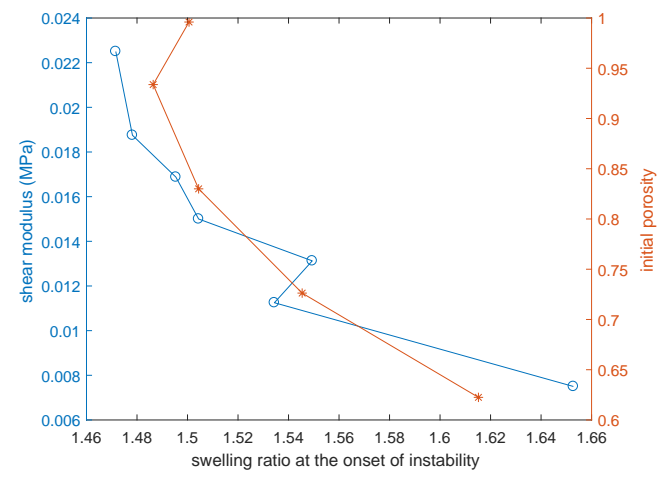

Fig. 20 Swelling ratios at the onset of instabilities with various shear modulus (blue line) and initial porosity (red line).

agreement with the experimental findings reported by Trujillo et al. ${ }^{32}$ (see Fig. 22), in which the sodium acrylate content (which determines the fixed charge density) and bisacrylamide (crosslinker) content of a poly (acrylamide-co-sodium acrylate) hydrogels (which determines the shear modulus) were varied.

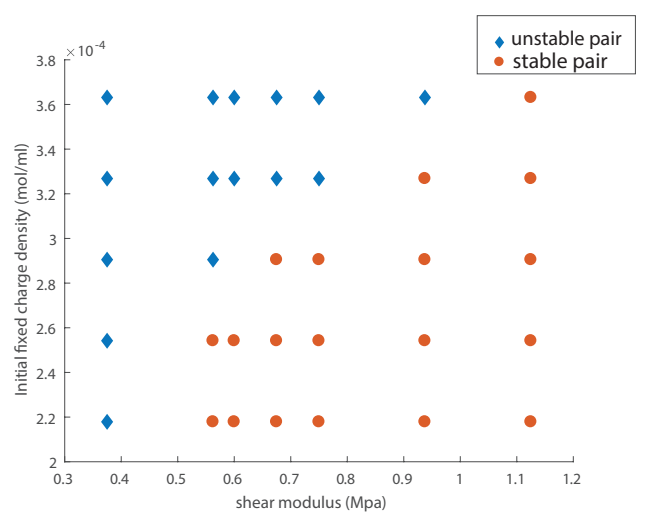

Fig. 21 Stability diagram indication stable/unstable pair of given shear modulus and fixed charge density. Red dot: stable, blue diamond: unstable.

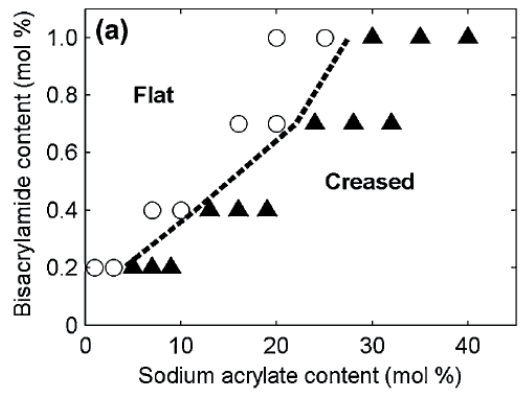

Fig. 22 Experimental results from Trujillo et al. ${ }^{32}$ : stability diagram indicating the region of composition space for which surface-attached poly(acrylamide-co-sodium acrylate) hydrogels form creases.

\subsection{Swelling of a gel tube in 3D}

This example is inspired by the experimental results reported by Lee et al. ${ }^{57}$. The schematic diagram of the gel tube geometry is given in Fig. 23. The geometry is featured by three parameters: diameter $D$, height $h$ and thickness $t$. One side of the tube is fixed and the other side is in touch with the other solution. Due to the geometry constraint, circumferential wrinkling might occur during swelling. According to Lee et al. ${ }^{57}$, the gel geometry (especially $h / D$ and $t / h$ ) has a dominant influence on the buckling of the swelling gel. Especially, a smaller normalized thickness $t / h$ implies a larger chance of instability and a smaller normalized height leads to a larger wave number in the buckling pattern.

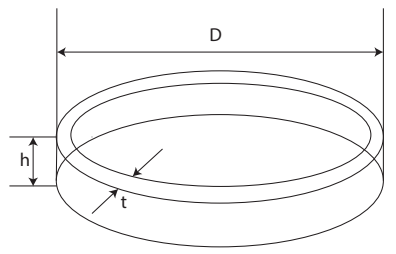

Fig. 23 Initial gel tube geometry illustration

Using proposed numerical model the scenario described above is simulated. We examine three sets of geometry parameters $(D, t, h)$ : "standard", "thick" and "tall" using the same time steps and mesh size assuming constant permeability. For the "standard" set, we set $(D, t, h)=(5,0.2,0.6)(\mathrm{mm})$. The chemical potential contour plot of the tubular gel at different moments is given in Fig. 24. We double the thickness and height of the "thick" and "tall" set. In other words, we have $(D, t, h)=(5,0.4,0.6)(\mathrm{mm})$ for the "thick" set (Fig. 25) and $(D, t, h)=(5,0.2,1.2)(\mathrm{mm})$ for the "tall" set (Fig. 26). The evolution of these chemical potential contour plots in time are included in ESI. We observe that in the standard set, buckling appeared as a wave-like pattern and ended up with 6 wrinkles. The "thick" set did not show any buckling at all and the "tall" set showed buckling but with fewer wrinkles (4 instead of 6). To sum up, our simulation results are in complete agreement with the experiment in terms of the relationship between the geometry and buckling behavior. Moreover, the buckling patterns observed in the simulations resemble the ones from the experiment.

At last, we investigate the influence of the strain-dependent permeability on the buckling behavior. In the "thick" set, instead of constant constant permeability, we apply the permeability law given in equation (25) with $\beta=1.5$. The chemical potential contours are plotted in Fig. 27. We see that the buckling appeared and with 5 winkles this time. This result suggests that the appearance of buckling is not only related to geometry but also to how fast the fluid permeates into the gel.

\section{Conclusions}

In the current work, we presented a numerical model to simulate the swelling of SAP hydrogel in three dimensions with arbitrary shape. The main swelling mechanism features Donnan osmosis instead of the mixing energy ${ }^{61,62}$ due to the existence of the fixed charge groups. The proposed numerical approach $(\mathrm{MH}-$ FEM) guarantees a more robust and accurate calculation of the 


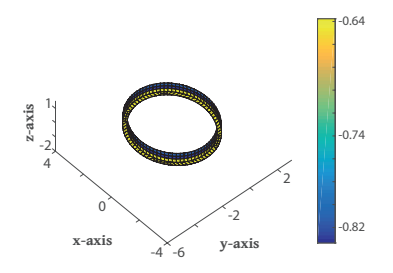

(a) $t=5 \mathrm{~s}$

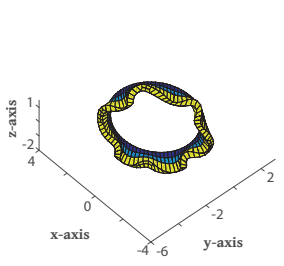

(b) $t=415 \mathrm{~s}$
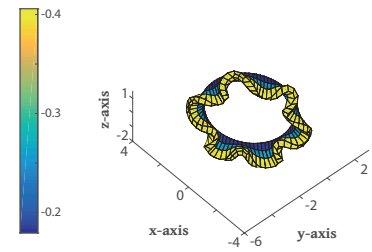

(c) t=830 s
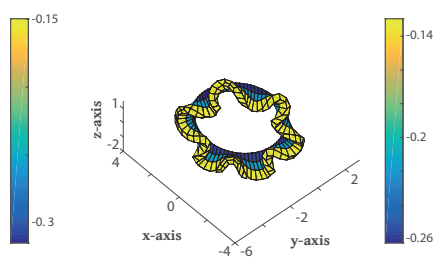

(d) $t=1250 \mathrm{~s}$

Fig. 24 Contour plots of chemical potential on the "standard" tubular gel with constant permeabilitiy at different moments

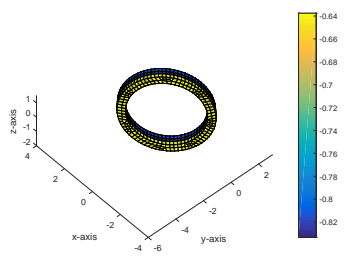

(a) $t=5 \mathrm{~s}$

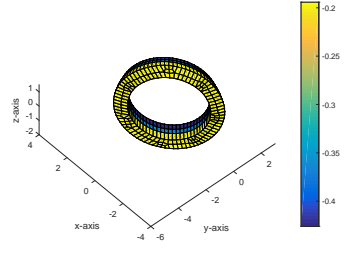

(b) $t=415 \mathrm{~s}$

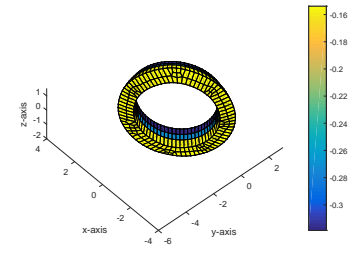

(c) $t=830 \mathrm{~s}$

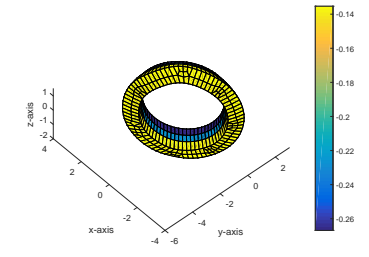

(d) $t=1250 \mathrm{~s}$

Fig. 25 Contour plots of chemical potential on the "thick" tubular gel with constant permeabilitiy at different moments

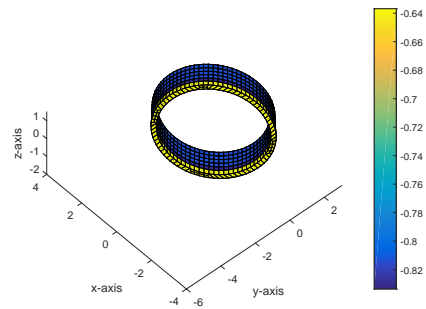

(a) $t=5 \mathrm{~s}$

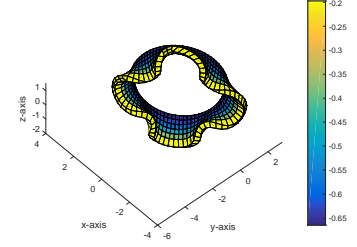

(b) $t=415 \mathrm{~s}$

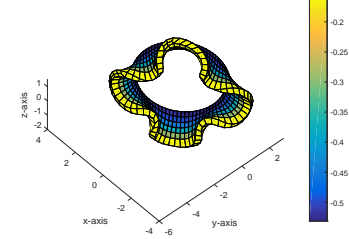

(c) $t=830 \mathrm{~s}$

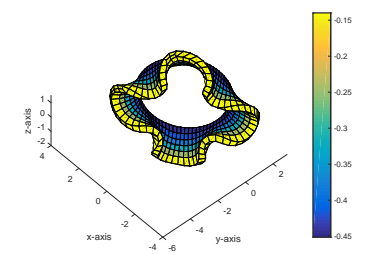

(d) t=1250 s

Fig. 26 Contour plots of chemical potential on the "tall" tubular gel with strain-dependent permeabilitiy at different moments

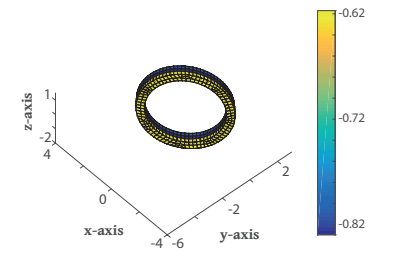

(a) $t=5 \mathrm{~s}$

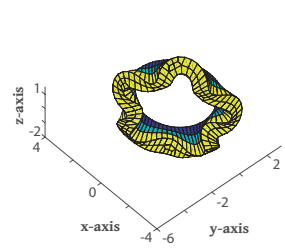

(b) $t=415 \mathrm{~s}$

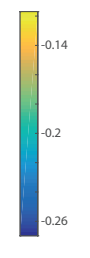

(c) $\mathrm{t}=830 \mathrm{~s}$
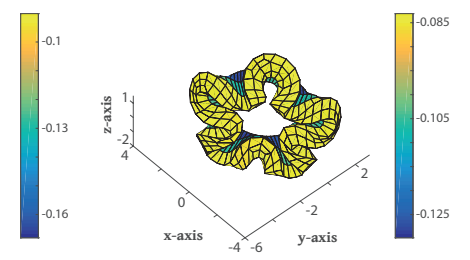

(d) $\mathrm{t}=1250 \mathrm{~s}$

Fig. 27 Contour plots of chemical potential on the "thick" tubular gel with strain-dependent permeabilitiy at different moments

flux field as well as the deformation field in transient swelling simulations, therefore, suitable for simulations that involve extremely large deformations.

Simulation results are first compared with analytical solutions of two classic poromechanical problems (Terzaghi's problem and Cryer's problem) in small deformations. Next, the results are compared with a benchmark solution calculated by MATLAB in one dimension. The good agreement between the two indicates the reliability of the model in the finite deformation regime. To demonstrate the capability of the numerical model, we presented three numerical examples: free swelling of a spherical gel, free swelling of a gel with realistic geometry and squeezing of a cubic gel. Maximal principal stress and chemical potential are shown in the form of contour plots to indicate the swelling state at a given moment.

As next step, we make use of the proposed numerical model to study the onset of the surface instability (typically bucklings). Specifically, the influence of the various parameters (mesh sizes and material parameters) on the onset of instabilities are investigated in a swelling two-dimensional thin gel slab scenario. The numerical model is able to capture a large (local) deformations generated by wrinkling until self-contact occurs. Note that all the surface instabilities we observed in the simulations resulted from transient swelling without any initial perturbation. As a conse- 
quence, it is not possible to do a straightforward comparison with the results we derived from the linear perturbation analysis at the equilibrium state. However, the swelling ratios we derived from the simulations are typically between 1.4 and 2.1 which is in accordance with the linear perturbation analysis and experimental results $^{32}$. The three dimensional swelling of a tubular gel under geometric constraints is another demonstration that the model is able to capture complex 3D buckling patterns. Moreover, the buckling patterns are qualitatively in accordance with the experimental findings.

To sum up, MHFEM model provides an alternative for modeling of swelling hydrogels. In the simulation involving extremely large deformations, MHFEM provides a more robust and accurate solution than the methods without the local mass conservation properties for the fluid transport. The surface instabilities simulation results are good demonstrations for the capabilities of the proposed numerical model.

\section{Acknowledgment}

The authors acknowledge the financial support from the Technology Foundation STW, the technological branch of the Netherlands Organization of Scientific Research and the Ministry of Economic Affairs (project number 12538).

\section{References}

1 T. Tanaka, Sci. Am., 1981, 244, 124.

2 S. Rimmer, Biomedical hydrogels: biochemistry, manufacture and medical applications, Elsevier, 2011.

3 M. J. Zohuriaan-Mehr and K. Kabiri, Iran. Polym. J., 2008, 17, 451.

4 A. S. Hoffman, Adv. Drug Delivery Rev., 2012, 64, 18-23.

5 A. Sannino, C. Demitri and M. Madaghiele, Materials, 2009, 2, 353-373.

6 F. L. Buchholz and A. T. Graham, John! Wiley \& Sons, Inc, 605 Third Ave, New York, NY 10016, USA, 1998. 279, 1998.

7 M. A. Biot, J. Appl. Phys., 1941, 12, 155-164.

8 W. Hong, X. Zhao, J. Zhou and Z. Suo, J. Mech. Phys. Solids, 2008, 56, 1779-1793.

9 S. A. Chester and L. Anand, J. Mech. Phys. Solids, 2010, 58, 1879-1906.

10 S. A. Chester, Soft Matter, 2012, 8, 8223-8233.

11 F. P. Duda, A. C. Souza and E. Fried, J. Mech. Phys. Solids, 2010, 58, 515-529.

12 P. J. Flory, Principles of polymer chemistry, Cornell University Press, 1953.

13 P. J. Flory and J. Rehner Jr, J. Chem. Phys., 1943, 11, 521526.

14 N. Bouklas and R. Huang, Soft Matter, 2012, 8, 8194-8203.

15 C. Truesdell and R. Toupin, Principles of Classical Mechanics and Field Theory/Prinzipien der Klassischen Mechanik und Feldtheorie, Springer, 1960, pp. 226-858.

16 R. M. Bowen, Int. J. Eng. Sci., 1980, 18, 1129-1148.

17 Y. Lanir, Biorheology, 1986, 24, 173-187.

18 W. M. Lai, J. S. Hou and V. C. Mow, J. Biomech. Eng., 1991,
113, 245-258.

19 J. M. Huyghe and J. D. Janssen, Int. J. Eng. Sci., 1997, 35, 793-802.

20 F. Lai and H. Li, Soft Matter, 2010, 6, 311-320.

21 T. Bertrand, J. Peixinho, S. Mukhopadhyay and C. W. MacMinn, Phys. Rev. Appl., 2016, 6, 064010.

22 R. Mosé, P. Siegel, P. h. Ackerer and G. Chavent, Water Resour. Res., 1994, 30, 3001-3012.

23 E. F. Kaasschieter and A. J. M. Huijben, Numer. Methods Partial Differ. Equ., 1992, 8, 221-266.

24 K. Malakpoor, E. F. Kaasschieter and J. M. Huyghe, ESAIM: Math. Modell. Numer. Anal., 2007, 41, 679-712.

25 S. Eran, M. Marder and H. L. Swinney, Am. Sci, 2004, 92, 254.

26 W. Wong, T. Guo, Y. Zhang and L. Cheng, Soft Matter, 2010, 6, 5743-5750.

27 S. Yang, K. Khare and P.-C. Lin, Adv. Funct. Mater., 2010, 20, 2550-2564.

28 T. Tanaka, S.-T. Sun, Y. Hirokawa, S. Katayama, J. Kucera, Y. Hirose and T. Amiya, Nature, 1987, 325, 796-798.

29 M. K. Kang and R. Huang, Soft Matter, 2010, 6, 5736-5742.

30 M. K. Kang and R. Huang, J. Mech. Phys. Solids, 2010, 58, 1582-1598.

31 Z. Wu, N. Bouklas and R. Huang, Int. J. Solids Struct., 2013, 50, 578-587.

32 V. Trujillo, J. Kim and R. C. Hayward, Soft Matter, 2008, 4, 564-569.

33 M. Guvendiren, J. A. Burdick and S. Yang, Soft Matter, 2010, 6, 2044-2049.

34 W. Toh, Z. Ding, T. Y. Ng and Z. Liu, J. Appl. Mech., 2015, 82, 061004.

35 M. K. Kang and R. Huang, J. Appl. Mech., 2010, 77, 061004.

36 J. Li, Y. Hu, J. J. Vlassak and Z. Suo, Soft Matter, 2012, 8, 8121-8128.

37 C. W. MacMinn, E. R. Dufresne and J. S. Wettlaufer, Phys. Rev. Appl., 2016, 5, 044020.

38 F. Horkay, I. Tasaki and P. J. Basser, Biomacromolecules, 2000, 1, 84-90.

39 R. C. Weast, M. J. Astle, W. H. Beyer et al., CRC handbook of chemistry and physics, CRC press Boca Raton, FL, 1988, vol. 69.

40 O. Coussy, Poromechanics, John Wiley \& Sons, 2004.

41 W. Wilson, J. M. Huyghe and C. C. Van Donkelaar, Biomech. Model. Mechanobiol., 2007, 6, 43-53.

42 A. Katchalsky and P. F. Curran, Nonequilibrium thermodynamics in biophysics, Harvard University Press, 1967.

43 J. Kozeny, Über kapillare leitung des wassers im boden:(aufstieg, versickerung und anwendung auf die bewässerung), Hölder-Pichler-Tempsky, 1927.

44 P. C. Carman, Transactions-Institution of Chemical Engineeres, 1937, 15, 150-166.

45 W. M. Lai and V. C. Mow, Biorheology, 1980, 17, 111.

46 A. Van der Voet, Proceedings of the Institution of Mechanical Engineers. Part H, Journal of engineering in medicine, 1997, 
211, 209.

47 W. Wilson, C. C. Van Donkelaar, R. Van Rietbergen and R. Huiskes, J. Biomed. Eng., 2005, 27, 810-826.

48 M. Tokita and T. Tanaka, J. Chem. Phys., 1991, 95, 46134619.

49 C. A. Grattoni, H. H. Al-Sharji, C. Yang, A. H. Muggeridge and R. W. Zimmerman, J. Colloid Interface Sci., 2001, 240, 601607.

50 M. Engelsberg and W. Barros Jr, Phys. Rev. E, 2013, 88, 062602.

51 F. Brezzi and M. Fortin, Mixed and hybrid finite element methods, Springer Science \& Business Media, 2012, vol. 15.

52 K. v. Terzaghi, Sitzungsberichte der Akademie der Wissenschaften in Wien, Mathematisch-Naturwissenschaftliche Klasse, Abteilung IIa, 1923, 132, 125-138.

53 K. Terzaghi et al., 1925.
54 C. Cryer, The Quarterly Journal of Mechanics and Applied Mathematics, 1963, 16, 401-412.

55 E. Holzbecher, Int. J. Multiphys., 2016, 10, year.

56 S. C. Tadepalli, A. Erdemir and P. R. Cavanagh, J. Biomech., 2011, 44, 2337-2343.

57 H. Lee, J. Zhang, H. Jiang and N. X. Fang, Phys. Rev. Lett., 2012, 108, 214304.

58 W. Hong, X. Zhao and Z. Suo, Applied Physics Letters, 2009, 95, 111901.

59 N. Bouklas, C. M. Landis and R. Huang, Journal of the Mechanics and Physics of Solids, 2015, 79, 21-43.

60 W. R. Drummond, M. L. Knight, M. L. Brannon and N. A. Peppas, Journal of controlled release, 1988, 7, 181-183.

61 P. J. Flory, J. Chem. Phys., 1942, 10, 51-61.

62 M. L. Huggins, J. Chem. Phys., 1941, 9, 440-440. 